\title{
Self-Assessing Photoluminescent Polyurethanes
}

\author{
Brent R. Crenshaw and Christoph Weder
}

Department of Macromolecular Science and Engineering, Case Western Reserve

University, 2100 Adelbert Road, Cleveland, Ohio 44106-7202

Supporting Information 


\section{Details for the melt polymerization of polymers MP1-MP5 and MP7}

MP1: PTMG (1.80 g, $1.80 \mathrm{mmol}), 1 \mathrm{c}(0.16 \mathrm{~g}, 0.20 \mathrm{mmol})$, MDI (0.52 g, $2.08 \mathrm{mmol})$. MP1 was obtained as an orange rubbery solid. ${ }^{1} \mathrm{H}$ NMR: $\delta=$ MDI residue: 9.52 (s, 2H, NH), 7.51 (d, 4H, ArH), 7.18 (d, 4H, ArH), 3.87 (s, 2H, $\left.\mathrm{CH}_{2}-\mathrm{Ar}\right)$; PTMG residue: 4.12 (t, 4H, $\left.\mathrm{CH}_{2}-\mathrm{OOC}\right), 3.39\left(\mathrm{~s}, 52 \mathrm{H}, \mathrm{CH}_{2}-\mathrm{O}\right), 1.69\left(\mathrm{~s}, 4 \mathrm{H}, \mathrm{CH}_{2}\right), 1.58\left(\mathrm{~s}, 52 \mathrm{H}, \mathrm{CH}_{2}\right) ; \mathbf{1 c}$ residue: $8.02(2 \mathrm{H}$, ArH obstructed $), 7.88(\mathrm{~s}, 2 \mathrm{H}, \mathrm{CH}=\mathrm{CCN}), 7.78(\mathrm{~d}, 4 \mathrm{H}, \mathrm{ArH}), 7.13$ (4 $\mathrm{H}$, ArH obstructed), 4.12 (4H, $\mathrm{CH}_{2}$-OOC obstructed), 4.11 (4H, $\mathrm{CH}_{2}-\mathrm{OAr}$ obstructed), $4.00\left(\mathrm{~s}, 6 \mathrm{H}, \mathrm{CH}_{3}-\mathrm{OAr}\right), 1.8\left(\mathrm{~m}, 4 \mathrm{H}, \mathrm{CH}_{2}\right), 1.5\left(\mathrm{~m}, 4 \mathrm{H}, \mathrm{CH}_{2}\right) 1.4-1.2(\mathrm{~m}, 32 \mathrm{H}, 16 \mathrm{x}$ $\mathrm{CH}_{2}$ ); end groups: 6.90 (d, 2H, $\left.\mathrm{ArH}\right), 6.60(\mathrm{~d}, 2 \mathrm{H}, \mathrm{ArH}), 4.97\left(\mathrm{~s}, 2 \mathrm{H}, \mathrm{NH}_{2}\right)$. Anal. Calcd for $\left(\mathrm{MDI}_{2.08} \mathrm{PTMG}_{1.80} \mathbf{1 c}_{0.20}\right)_{\mathrm{n}}: \mathrm{C}, 67.45 ; \mathrm{H}, 9.52 ; \mathrm{N}, 2.57$. Found: C, 66.84; H, 9.86; N, 2.51

MP2: PTMG (1.80 g, $1.80 \mathrm{mmol})$, 1c (0.16 g, $0.20 \mathrm{mmol})$, BDO (0.09 g, $0.96 \mathrm{mmol})$, MDI (0.77 g, $3.07 \mathrm{mmol})$. MP2 was obtained as an orange rubbery solid. ${ }^{1} \mathrm{H}$ NMR: $\delta=$ MDI residue: 9.55 (s, 2H, NH), 9.52 (s, 2H, NH), 7.51 (d, 4H, ArH), 7.17 (d, 4H, ArH), 3.87 (s, 2H, $\left.\mathrm{CH}_{2}-\mathrm{Ar}\right)$; PTMG residue: 4.12 (t, 4H, $\left.\mathrm{CH}_{2}-\mathrm{OOC}\right), 3.39$ (s, 52H, $\left.\mathrm{CH}_{2}-\mathrm{O}\right)$, 1.69 (s, 4H, $\left.\mathrm{CH}_{2}\right), 1.58\left(\mathrm{~s}, 52 \mathrm{H}, \mathrm{CH}_{2}\right)$; BDO residue: 4.13 (d, 4H, $\left.\mathrm{CH}_{2}-\mathrm{O}\right), 1.73(\mathrm{~s}, 4 \mathrm{H}$, $\mathrm{CH}_{2}$ ); 1c residue: 8.02 (2H, ArH obstructed), 7.88 (s, $\left.2 \mathrm{H}, \mathrm{CH}=\mathrm{CCN}\right), 7.78(\mathrm{~d}, 4 \mathrm{H}$, ArH), 7.13 (4 H, ArH obstructed), 4.12 (4H, $\mathrm{CH}_{2}-\mathrm{OOC}$ obstructed), 4.11 (4H, $\mathrm{CH}_{2}$-OAr obstructed), 4.00 (s, $\left.6 \mathrm{H}, \mathrm{CH}_{3}-\mathrm{OAr}\right), 1.8\left(\mathrm{~m}, 4 \mathrm{H}, \mathrm{CH}_{2}\right), 1.5\left(\mathrm{~m}, 4 \mathrm{H}, \mathrm{CH}_{2}\right) 1.4$ - $1.2\left(\mathrm{~m}, 32 \mathrm{H}, 16 \times \mathrm{CH}_{2}\right)$; end groups: 6.90 (d, 2H, $\left.\mathrm{ArH}\right), 6.60(\mathrm{~d}, 2 \mathrm{H}, \mathrm{ArH}), 4.97(\mathrm{~s}, 2 \mathrm{H}$, $\left.\mathrm{NH}_{2}\right)$. Anal. Calcd for $\left(\mathrm{MDI}_{3.07} \mathrm{PTMG}_{1.80} \mathrm{BDO}_{0.96} \mathbf{1 c}_{0.20}\right)_{\mathrm{n}}$ : C, 67.42; H, 9.08; N, 3.26. Found: C, 67.37; H, 9.44; N, 3.35. 
MP3: PTMG (1.80 g, $1.80 \mathrm{mmol}), 1 \mathrm{c}(0.16 \mathrm{~g}, 0.20 \mathrm{mmol}), \mathrm{BDO}(0.17 \mathrm{~g}, 1.90 \mathrm{mmol})$, MDI (1.01 g, $4.05 \mathrm{mmol})$. MP3 was obtained as an orange rubbery solid. ${ }^{1} \mathrm{H}$ NMR: $\delta=$ MDI residue: 9.55 (s, 2H, NH), 9.52 (s, 2H, NH), 7.51 (d, 4H, ArH), 7.17 (d, 4H, ArH), 3.87 (s, 2H, $\mathrm{CH}_{2}-\mathrm{Ar}$ ); PTMG residue: 4.12 (t, $\left.4 \mathrm{H}, \mathrm{CH}_{2}-\mathrm{OOC}\right), 3.39$ (s, $52 \mathrm{H}, \mathrm{CH}_{2}-\mathrm{O}$ ), $1.69\left(\mathrm{~s}, 4 \mathrm{H}, \mathrm{CH}_{2}\right), 1.58\left(\mathrm{~s}, 52 \mathrm{H}, \mathrm{CH}_{2}\right)$; $\mathrm{BDO}$ residue: $4.13\left(\mathrm{~d}, 4 \mathrm{H}, \mathrm{CH}_{2}-\mathrm{O}\right), 1.73$ (s, $4 \mathrm{H}$, $\left.\mathrm{CH}_{2}\right)$; 1c residue: $8.02(2 \mathrm{H}$, ArH obstructed $), 7.88(\mathrm{~s}, 2 \mathrm{H}, \mathrm{CH}=\mathrm{CCN}), 7.78(\mathrm{~d}, 4 \mathrm{H}$, ArH), $7.13(4 \mathrm{H}$, ArH obstructed $), \sim 4.12\left(4 \mathrm{H}, \mathrm{CH}_{2}-\mathrm{OOC}\right.$ obstructed $), 4.11(4 \mathrm{H}$, CH $\mathrm{CH}_{2}-\mathrm{OAr}$ obstructed), 4.00 (s, $\left.6 \mathrm{H}, \mathrm{CH}_{3}-\mathrm{OAr}\right), 1.8\left(\mathrm{~m}, 4 \mathrm{H}, \mathrm{CH}_{2}\right), 1.5\left(\mathrm{~m}, 4 \mathrm{H}, \mathrm{CH}_{2}\right) 1.4$ - $1.2\left(\mathrm{~m}, 32 \mathrm{H}, 16 \times \mathrm{CH}_{2}\right)$; end groups: $6.90(\mathrm{~d}, 2 \mathrm{H}, \mathrm{ArH}), 6.60(\mathrm{~d}, 2 \mathrm{H}, \mathrm{ArH}), 4.97(\mathrm{~s}, 2 \mathrm{H}$, $\left.\mathrm{NH}_{2}\right)$. Anal. Calcd for $\left(\mathrm{MDI}_{4.05} \mathrm{PTMG}_{1.80} \mathrm{BDO}_{1.90} \mathbf{1 c}_{0.20}\right)_{\mathrm{n}}: \mathrm{C}, 67.40 ; \mathrm{H}, 8.74 ; \mathrm{N}, 3.79$. Found: $\mathrm{C}, 67.60 ; \mathrm{H}, 9.21 ; \mathrm{N}, 3.98$.

MP4: PTMG (1.80 g, $1.80 \mathrm{mmol}), 1 \mathrm{c}(0.16 \mathrm{~g}, 0.20 \mathrm{mmol})$, BDO (0.26 g, $2.83 \mathrm{mmol})$, MDI (1.26 g, $5.02 \mathrm{mmol})$. MP4 was obtained as an orange rubbery solid. ${ }^{1} \mathrm{H}$ NMR: $\delta=$ MDI residue: 9.55 (s, 2H, NH), 9.52 (s, 2H, NH), 7.51 (d, 4H, ArH), 7.17 (d, 4H, ArH), 3.87 (s, 2H, $\mathrm{CH}_{2}-\mathrm{Ar}$ ); PTMG residue: 4.12 (t, $\left.4 \mathrm{H}, \mathrm{CH}_{2}-\mathrm{OOC}\right), 3.39$ (s, 52H, $\mathrm{CH}_{2}-\mathrm{O}$ ), $1.69\left(\mathrm{~s}, 4 \mathrm{H}, \mathrm{CH}_{2}\right), 1.58\left(\mathrm{~s}, 52 \mathrm{H}, \mathrm{CH}_{2}\right)$; $\mathrm{BDO}$ residue: $4.13\left(\mathrm{~d}, 4 \mathrm{H}, \mathrm{CH}_{2}-\mathrm{O}\right), 1.73(\mathrm{~s}, 4 \mathrm{H}$, $\left.\mathrm{CH}_{2}\right)$; 1c residue: $8.02(2 \mathrm{H}$, ArH obstructed $), 7.88(\mathrm{~s}, 2 \mathrm{H}, \mathrm{CH}=\mathrm{CCN}), 7.78(\mathrm{~d}, 4 \mathrm{H}$, ArH), $7.13(4 \mathrm{H}$, ArH obstructed $), \sim 4.12\left(4 \mathrm{H}, \mathrm{CH}_{2}-\mathrm{OOC}\right.$ obstructed $), 4.11(4 \mathrm{H}$, $\mathrm{CH}_{2}-\mathrm{OAr}$ obstructed), 4.00 (s, $\left.6 \mathrm{H}, \mathrm{CH}_{3}-\mathrm{OAr}\right), 1.8\left(\mathrm{~m}, 4 \mathrm{H}, \mathrm{CH}_{2}\right), 1.5\left(\mathrm{~m}, 4 \mathrm{H}, \mathrm{CH}_{2}\right) 1.4$ - $1.2\left(\mathrm{~m}, 32 \mathrm{H}, 16 \times \mathrm{CH}_{2}\right)$; end groups: $6.90(\mathrm{~d}, 2 \mathrm{H}, \mathrm{ArH}), 6.60(\mathrm{~d}, 2 \mathrm{H}, \mathrm{ArH}), 4.97(\mathrm{~s}, 2 \mathrm{H}$, $\mathrm{NH}_{2}$ ). Anal. Calcd for $\left(\mathrm{MDI}_{5.02} \mathrm{PTMG}_{1.80} \mathrm{BDO}_{2.83} \mathbf{1 c}_{0.20}\right)_{\mathrm{n}}: \mathrm{C}, 67.39 ; \mathrm{H}, 8.47 ; \mathrm{N}, 4.22$. Found: $\mathrm{C}, 67.41 ; \mathrm{H}, 8.89 ; \mathrm{N}, 4.21$. 
MP5: PTMG (1.80 g, $1.80 \mathrm{mmol}), 1 \mathrm{c}(0.16 \mathrm{~g}, 0.20 \mathrm{mmol}), \mathrm{BDO}(0.37 \mathrm{~g}, 4.14 \mathrm{mmol})$, MDI (1.60 g, $6.38 \mathrm{mmol})$. MP5 was obtained as an orange rubbery solid. ${ }^{1} \mathrm{H}$ NMR: $\delta=$ MDI residue: 9.55 (s, 2H, NH), 9.52 (s, 2H, NH), 7.51 (d, 4H, ArH), 7.17 (d, 4H, ArH), 3.87 (s, 2H, $\mathrm{CH}_{2}-\mathrm{Ar}$ ); PTMG residue: 4.12 (t, $\left.4 \mathrm{H}, \mathrm{CH}_{2}-\mathrm{OOC}\right), 3.39$ (s, $52 \mathrm{H}, \mathrm{CH}_{2}-\mathrm{O}$ ), $1.69\left(\mathrm{~s}, 4 \mathrm{H}, \mathrm{CH}_{2}\right), 1.58\left(\mathrm{~s}, 52 \mathrm{H}, \mathrm{CH}_{2}\right)$; $\mathrm{BDO}$ residue: $4.13\left(\mathrm{~d}, 4 \mathrm{H}, \mathrm{CH}_{2}-\mathrm{O}\right), 1.73$ (s, $4 \mathrm{H}$, $\left.\mathrm{CH}_{2}\right)$; 1c residue: $8.02(2 \mathrm{H}$, ArH obstructed $), 7.88(\mathrm{~s}, 2 \mathrm{H}, \mathrm{CH}=\mathrm{CCN}), 7.78(\mathrm{~d}, 4 \mathrm{H}$, ArH), $7.13(4 \mathrm{H}$, ArH obstructed $), \sim 4.12\left(4 \mathrm{H}, \mathrm{CH}_{2}-\mathrm{OOC}\right.$ obstructed $), 4.11(4 \mathrm{H}$, $\mathrm{CH}_{2}-\mathrm{OAr}$ obstructed), 4.00 (s, $\left.6 \mathrm{H}, \mathrm{CH}_{3}-\mathrm{OAr}\right), 1.8\left(\mathrm{~m}, 4 \mathrm{H}, \mathrm{CH}_{2}\right), 1.5\left(\mathrm{~m}, 4 \mathrm{H}, \mathrm{CH}_{2}\right) 1.4$ - $1.2\left(\mathrm{~m}, 32 \mathrm{H}, 16 \times \mathrm{CH}_{2}\right)$; end groups: $6.90(\mathrm{~d}, 2 \mathrm{H}, \mathrm{ArH}), 6.60(\mathrm{~d}, 2 \mathrm{H}, \mathrm{ArH}), 4.97(\mathrm{~s}, 2 \mathrm{H}$, $\left.\mathrm{NH}_{2}\right)$. Anal. Calcd for $\left(\mathrm{MDI}_{6.38} \mathrm{PTMG}_{1.8} \mathrm{BDO}_{4.14} \mathbf{1 c}_{0.2}\right)_{\mathrm{n}}$ : C, 67.37; H, 8.17; N, 4.70. Found: $\mathrm{C}, 67.26 ; \mathrm{H}, 8.58 ; \mathrm{N}, 4.80$.

MP7: PTMG (5.00 g, $5.00 \mathrm{mmol})$, BDO (0.65 g, $7.25 \mathrm{mmol})$, MDI (3.18 g, 12.70 mmol). MP7 was obtained as a clear rubbery solid. ${ }^{1} \mathrm{H}$ NMR: $\delta=$ MDI residue: 9.55 (s, 2H, NH), 9.52 (s, 2H, NH), 7.51 (d, 4H, ArH), 7.17 (d, 4H, ArH), 3.87 (s, 2H, $\left.\mathrm{CH}_{2}-\mathrm{Ar}\right)$; PTMG residue: 4.12 (t, 4H, $\left.\mathrm{CH}_{2}-\mathrm{OOC}\right), 3.39$ (s, 52H, $\left.\mathrm{CH}_{2}-\mathrm{O}\right), 1.69$ (s, 4H, $\left.\mathrm{CH}_{2}\right), 1.58$ (s, 52H, $\mathrm{CH}_{2}$ ); $\mathrm{BDO}$ residue: 4.13 (d, $\left.4 \mathrm{H}, \mathrm{CH}_{2}-\mathrm{O}\right), 1.73$ (s, $4 \mathrm{H}, \mathrm{CH}_{2}$ ); end groups: 6.90 $(\mathrm{d}, 2 \mathrm{H}, \quad \operatorname{ArH}), 6.60 \quad(\mathrm{~d}, 2 \mathrm{H}, \quad \mathrm{ArH}), 4.97\left(\mathrm{~s}, 2 \mathrm{H}, \quad \mathrm{NH}_{2}\right)$. Anal. Calcd for $\left(\mathrm{MDI}_{12.70} \mathrm{PTMG}_{5.00} \mathrm{BDO}_{7.25}\right)_{\mathrm{n}}: \mathrm{C}, 66.89 ; \mathrm{H}, 8.61 ; \mathrm{N}, 4.03$. Found: $\mathrm{C}, 66.72 ; \mathrm{H}, 9.07 ; \mathrm{N}$, 4.07 . 


\section{Details for the solution polymerization of polymers SP1-SP3 and SP5}

SP1: PTMG (1.122 g, $1.122 \mathrm{mmol}), 1 \mathrm{c}(0.067 \mathrm{~g}, 0.085 \mathrm{mmol})$, MDI (0.317 g, 1.266 mmol), DBTDL (0.015 g, $0.024 \mathrm{mmol})$. SP1 was obtained as an orange rubbery solid. ${ }^{1} \mathrm{H}$ NMR: $\delta=$ MDI residue: $9.52(\mathrm{~s}, 2 \mathrm{H}, \mathrm{NH}), 7.51(\mathrm{~d}, 4 \mathrm{H}, \mathrm{ArH}), 7.18(\mathrm{~d}, 4 \mathrm{H}, \mathrm{ArH}), 3.87$ (s, 2H, $\mathrm{CH}_{2}-\mathrm{Ar}$ ); PTMG residue: 4.12 (t, 4H, $\left.\mathrm{CH}_{2}-\mathrm{OOC}\right), 3.39$ (s, 52H, $\mathrm{CH}_{2}-\mathrm{O}$ ), 1.69 (s, 4H, $\left.\mathrm{CH}_{2}\right), 1.58$ (s, 52H, $\mathrm{CH}_{2}$ ); 1c residue: 8.02 (2H, ArH obstructed), 7.88 (s, $2 \mathrm{H}$, $\mathrm{CH}=\mathrm{CCN}), 7.78(\mathrm{~d}, 4 \mathrm{H}, \mathrm{ArH}), 7.13(4 \mathrm{H}, \mathrm{ArH}$ obstructed $), \sim 4.12\left(4 \mathrm{H}, \mathrm{CH}_{2}-\mathrm{OOC}\right.$ obstructed), 4.11 (4H, $\mathrm{CH}_{2}-\mathrm{OAr}$ obstructed), 4.00 (s, $\left.6 \mathrm{H}, \mathrm{CH}_{3}-\mathrm{OAr}\right), 1.8$ (m, $4 \mathrm{H}, \mathrm{CH}_{2}$ ), $1.5\left(\mathrm{~m}, 4 \mathrm{H}, \mathrm{CH}_{2}\right) 1.4-1.2\left(\mathrm{~m}, 32 \mathrm{H}, 16 \times \mathrm{CH}_{2}\right)$; end groups: 6.90 (d, 2H, $\left.\mathrm{ArH}\right), 6.60$ (d, $2 \mathrm{H}, \mathrm{ArH}), 4.97\left(\mathrm{~s}, 2 \mathrm{H}, \mathrm{NH}_{2}\right)$. Anal. Calcd for $\left(\mathrm{MDI}_{1.266} \mathrm{PTMG}_{1.122} \mathbf{1 c}_{0.085}\right)_{\mathrm{n}}: \mathrm{C}, 67.26 ; \mathrm{H}$, 9.57; N, 2.5. Found: C, 66.44; H, 10.16; N, 2.46.

SP2: PTMG (1.122 g, $1.122 \mathrm{mmol}), 1 c(0.089$ g, $0.112 \mathrm{mmol})$, BDO (0.112 g, 1.239 mmol), MDI (0.642 g, $2.564 \mathrm{mmol})$, DBTDL (0.016 g, $0.026 \mathrm{mmol})$. SP2 was obtained as an orange rubbery solid. ${ }^{1} \mathrm{H}$ NMR: $\delta=$ MDI residue: $9.55(\mathrm{~s}, 2 \mathrm{H}, \mathrm{NH}), 9.52(\mathrm{~s}, 2 \mathrm{H}$, NH), 7.51 (d, 4H, ArH), 7.17 (d, 4H, ArH), 3.87 (s, 2H, $\left.\mathrm{CH}_{2}-\mathrm{Ar}\right)$; PTMG residue: 4.12 (t, 4H, $\left.\mathrm{CH}_{2}-\mathrm{OOC}\right), 3.39$ (s, 52H, $\left.\mathrm{CH}_{2}-\mathrm{O}\right), 1.69$ (s, 4H, $\left.\mathrm{CH}_{2}\right), 1.58\left(\mathrm{~s}, 52 \mathrm{H}, \mathrm{CH}_{2}\right)$; BDO residue: $4.13\left(\mathrm{~d}, 4 \mathrm{H}, \mathrm{CH}_{2}-\mathrm{O}\right), 1.73\left(\mathrm{~s}, 4 \mathrm{H}, \mathrm{CH}_{2}\right)$; 1c residue: 8.02 (2H, ArH obstructed), $7.88(\mathrm{~s}, 2 \mathrm{H}, \mathrm{CH}=\mathrm{CCN}), 7.78(\mathrm{~d}, 4 \mathrm{H}, \mathrm{ArH}), 7.13(4 \mathrm{H}, \mathrm{ArH}$ obstructed $), ~ 4.12(4 \mathrm{H}$, $\mathrm{CH}_{2}$-OOC obstructed), 4.11 (4H, $\mathrm{CH}_{2}-\mathrm{OAr}$ obstructed), 4.00 (s, $6 \mathrm{H}, \mathrm{CH}_{3}-\mathrm{OAr}$ ), 1.8 (m, $\left.4 \mathrm{H}, \mathrm{CH}_{2}\right), 1.5\left(\mathrm{~m}, 4 \mathrm{H}, \mathrm{CH}_{2}\right) 1.4-1.2\left(\mathrm{~m}, 32 \mathrm{H}, 16 \mathrm{x} \mathrm{CH}_{2}\right)$; end groups: 6.90 (d, 2H, ArH $), \quad 6.60 \quad(\mathrm{~d}, \quad 2 \mathrm{H}, \quad \mathrm{ArH}), \quad 4.97 \quad\left(\mathrm{~s}, \quad 2 \mathrm{H}, \quad \mathrm{NH}_{2}\right) . \quad$ Anal. Calcd for $\left(\mathrm{MDI}_{2.564} \mathrm{PTMG}_{1.122} \mathrm{BDO}_{1.239} \mathbf{1 c}_{0.112}\right)_{\mathrm{n}}: \mathrm{C}, 67.35 ; \mathrm{H}, 8.73 ; \mathrm{N}, 3.82$. Found: C, 67.03; H, $9.14 ; \mathrm{N}, 3.72$. 
SP3: PTMG (1.125 g, $1.125 \mathrm{mmol}), 1 \mathrm{c}(0.099 \mathrm{~g}, 0.125 \mathrm{mmol})$, BDO (0.174 g, 1.930 mmol), MDI (0.812 g, $3.247 \mathrm{mmol})$, DBTDL (0.021 g, $0.033 \mathrm{mmol})$. SP3 was obtained as an orange rubbery solid. ${ }^{1} \mathrm{H}$ NMR: $\delta=$ MDI residue: $9.55(\mathrm{~s}, 2 \mathrm{H}, \mathrm{NH}), 9.52(\mathrm{~s}, 2 \mathrm{H}$, NH), 7.51 (d, 4H, ArH), 7.17 (d, 4H, ArH), 3.87 (s, 2H, $\mathrm{CH}_{2}-\mathrm{Ar}$ ); PTMG residue: 4.12 (t, 4H, $\left.\mathrm{CH}_{2}-\mathrm{OOC}\right), 3.39$ (s, 52H, $\left.\mathrm{CH}_{2}-\mathrm{O}\right), 1.69$ (s, 4H, $\left.\mathrm{CH}_{2}\right), 1.58$ (s, 52H, $\left.\mathrm{CH}_{2}\right)$; $\mathrm{BDO}$ residue: 4.13 (d, 4H, $\left.\mathrm{CH}_{2}-\mathrm{O}\right), 1.73$ (s, $\left.4 \mathrm{H}, \mathrm{CH}_{2}\right)$; 1 c residue: 8.02 (2H, ArH obstructed), $7.88(\mathrm{~s}, 2 \mathrm{H}, \mathrm{CH}=\mathrm{CCN}), 7.78(\mathrm{~d}, 4 \mathrm{H}, \mathrm{ArH}), 7.13(4 \mathrm{H}, \mathrm{ArH}$ obstructed $), \sim 4.12(4 \mathrm{H}$, $\mathrm{CH}_{2}-\mathrm{OOC}$ obstructed), 4.11 (4H, $\mathrm{CH}_{2}-\mathrm{OAr}$ obstructed), 4.00 (s, $\left.6 \mathrm{H}, \mathrm{CH}_{3}-\mathrm{OAr}\right), 1.8$ (m, $\left.4 \mathrm{H}, \mathrm{CH}_{2}\right), 1.5\left(\mathrm{~m}, 4 \mathrm{H}, \mathrm{CH}_{2}\right) 1.4-1.2\left(\mathrm{~m}, 32 \mathrm{H}, 16 \times \mathrm{CH}_{2}\right)$; end groups: $6.90(\mathrm{~d}, 2 \mathrm{H}$, ArH $), \quad 6.60 \quad(\mathrm{~d}, \quad 2 \mathrm{H}, \quad \mathrm{ArH}), \quad 4.97 \quad\left(\mathrm{~s}, \quad 2 \mathrm{H}, \quad \mathrm{NH}_{2}\right) . \quad$ Anal. Calcd for $\left(\mathrm{MDI}_{3.247} \mathrm{PTMG}_{1.125} \mathrm{BDO}_{1.930} \mathbf{1}_{0.125}\right)_{\mathrm{n}}: \mathrm{C}, 67.38 ; \mathrm{H}, 8.43 ; \mathrm{N}, 4.28$. Found: $\mathrm{C}, 66.62 ; \mathrm{H}$, $8.33 ; \mathrm{N}, 4.28$.

SP5: PTMG (2.502 g, 2.502 mmol), BDO (0.328 g, 3.643 mmol), MDI (1.584 g, 6.329 mmol), DBTDL (0.051 g, $0.080 \mathrm{mmol})$. SP5 was obtained as an off-white rubbery solid. ${ }^{1} \mathrm{H}$ NMR: $\delta$ = MDI residue: 9.55 (s, 2H, NH), 9.52 (s, 2H, NH), 7.51 (d, 4H, ArH), 7.17 (d, 4H, ArH), 3.87 (s, 2H, $\mathrm{CH}_{2}-\mathrm{Ar}$ ); PTMG residue: 4.12 (t, 4H, $\mathrm{CH}_{2}-\mathrm{OOC}$ ), 3.39 (s, 52H, $\mathrm{CH}_{2}-\mathrm{O}$ ), 1.69 (s, 4H, $\left.\mathrm{CH}_{2}\right), 1.58$ (s, 52H, $\mathrm{CH}_{2}$ ); $\mathrm{BDO}$ residue: $4.13\left(\mathrm{~d}, 4 \mathrm{H}, \mathrm{CH}_{2}-\mathrm{O}\right.$ ), $1.73\left(\mathrm{~s}, 4 \mathrm{H}, \mathrm{CH}_{2}\right)$; end groups: $6.90(\mathrm{~d}, 2 \mathrm{H}, \mathrm{ArH}), 6.60(\mathrm{~d}, 2 \mathrm{H}, \mathrm{ArH}), 4.97\left(\mathrm{~s}, 2 \mathrm{H}, \mathrm{NH}_{2}\right)$. Anal. Calcd for $\left(\mathrm{MDI}_{6.329} \mathrm{PTMG}_{2.502} \mathrm{BDO}_{3.643}\right)_{\mathrm{n}}: \mathrm{C}, 66.88 ; \mathrm{H}, 8.61 ; \mathrm{N}, 4.02$. Found: $\mathrm{C}$, 65.60; H, 8.46; N, 3.92. 


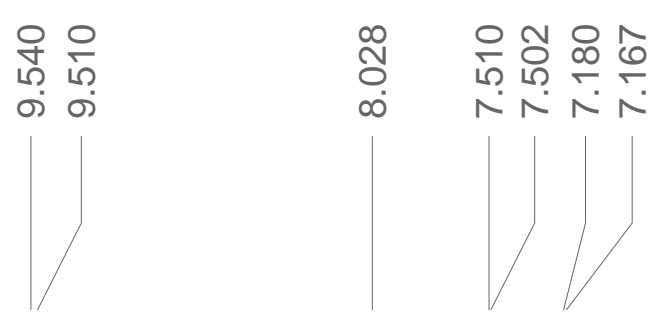

Figure S1: $600 \mathrm{MHz}{ }^{1} \mathrm{H}$ NMR Spectrum of Texin 985 in DMF- $\mathrm{d}_{6}$.

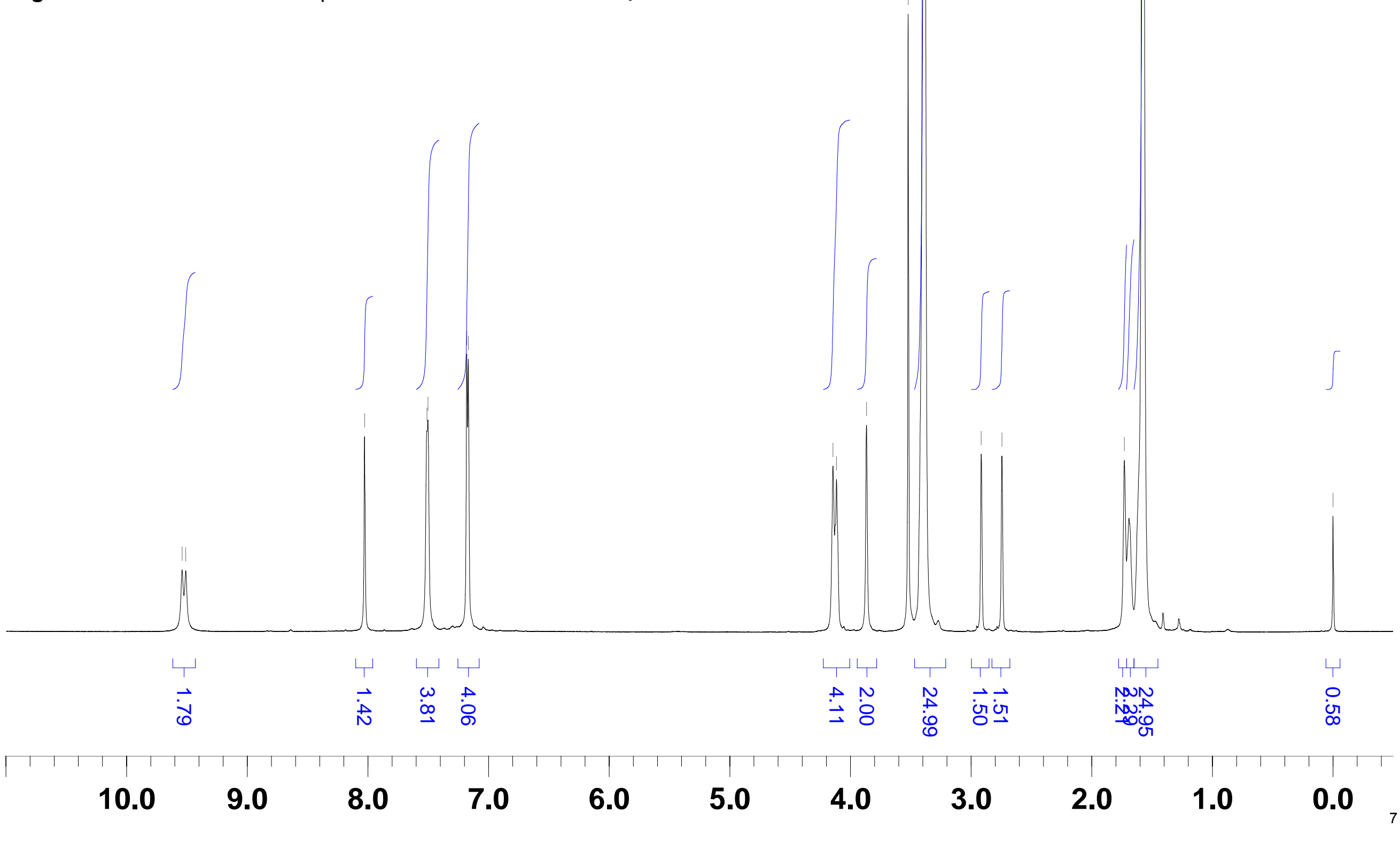



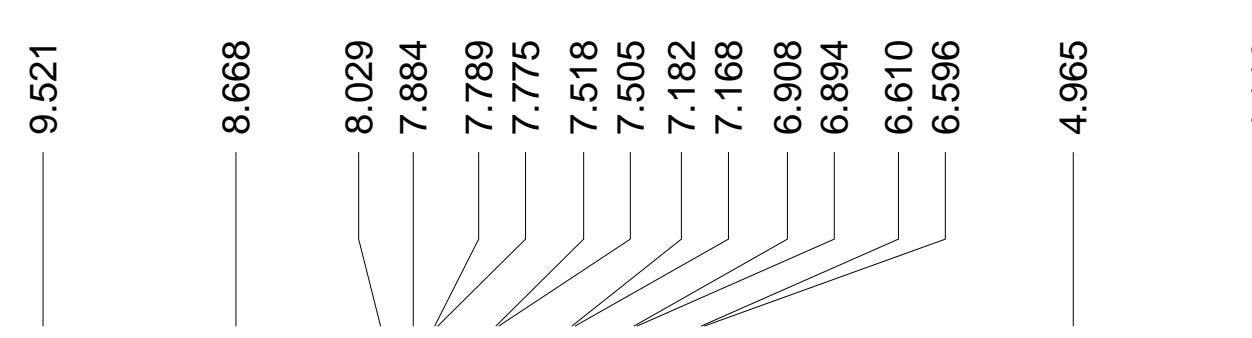

Figure S2: $600 \mathrm{MHz}{ }^{1} \mathrm{H}$ NMR Spectrum of MP1 in DMF- $\mathrm{d}_{6}$.

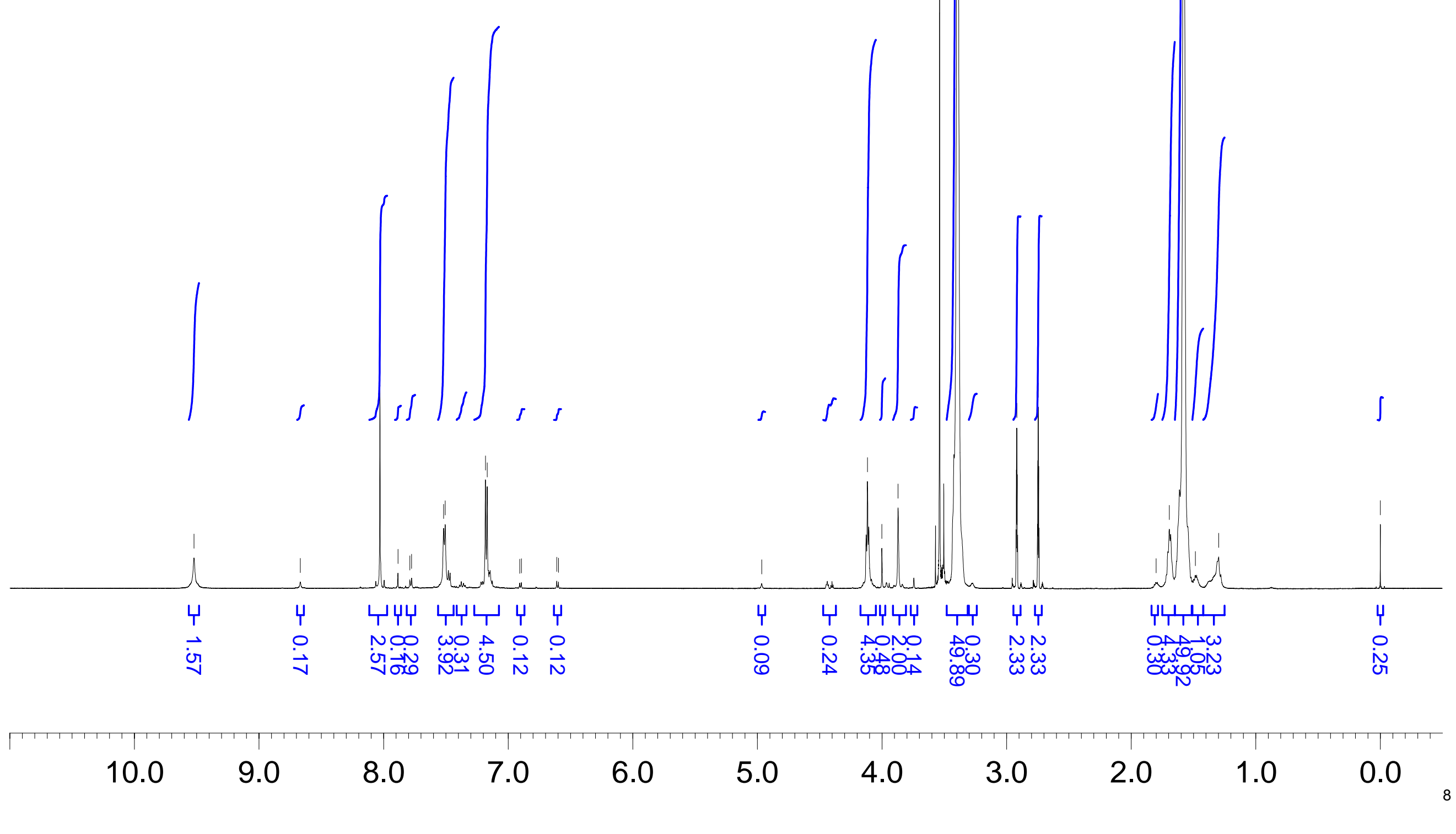

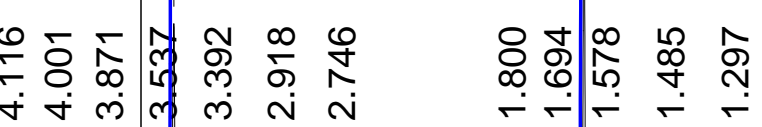

8
8
0 
Figure S3: $600 \mathrm{MHz}{ }^{1} \mathrm{H}$ NMR Spectrum of MP2 in DMF- $\mathrm{d}_{6}$.
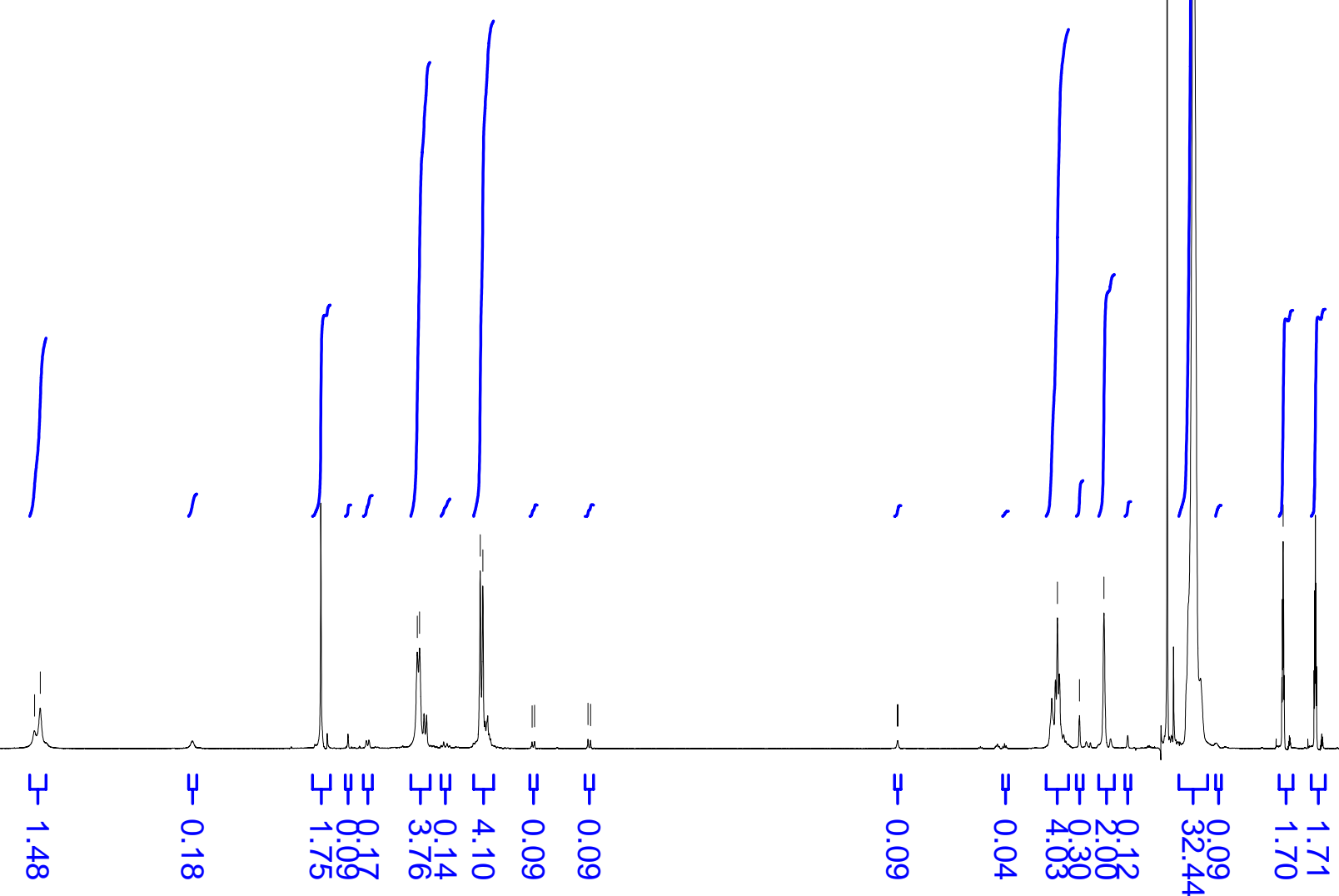


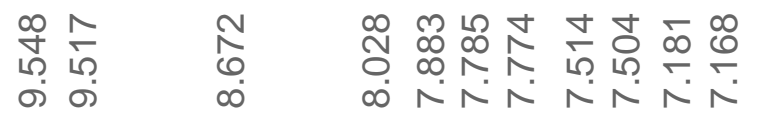

Figure S4: $600 \mathrm{MHz}{ }^{1} \mathrm{H}$ NMR Spectrum of MP3 in DMF- $\mathrm{d}_{6}$.

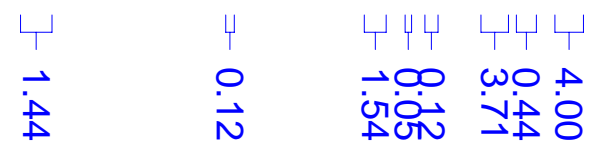

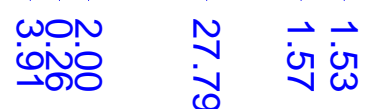

$\begin{array}{lll}\omega & N & \sim \\ \omega & \sim & \ddots\end{array}$

$\stackrel{\circ}{\mapsto}$

\section{0}

9.0

8.0

7.0

6.0

5.0

4.0

3.0

2.0

1.0

0.0 


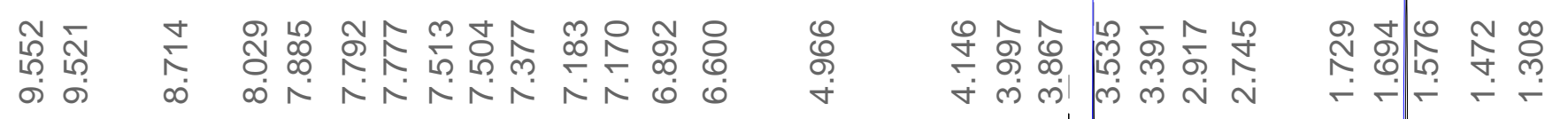

Figure S5: $600 \mathrm{MHz}{ }^{1} \mathrm{H}$ NMR Spectrum of MP4 in DMF- $\mathrm{d}_{6}$.

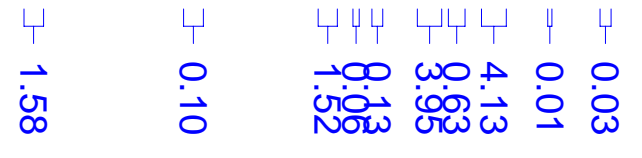

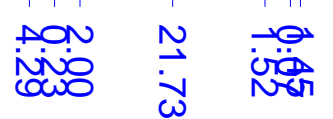

$\begin{array}{ll}\tilde{O} & 0 \\ \stackrel{0}{\omega} & \stackrel{0}{ } \\ 0 & \end{array}$

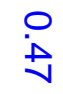

11.010 .0 


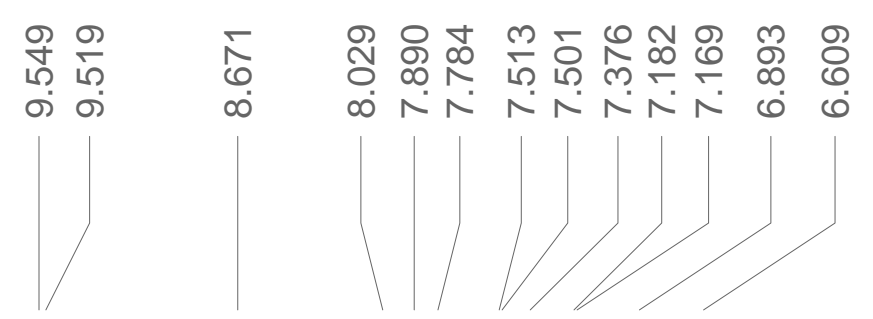

Figure S6: $600 \mathrm{MHz}{ }^{1} \mathrm{H}$ NMR Spectrum of MP5 in DMF-d ${ }_{6}$.
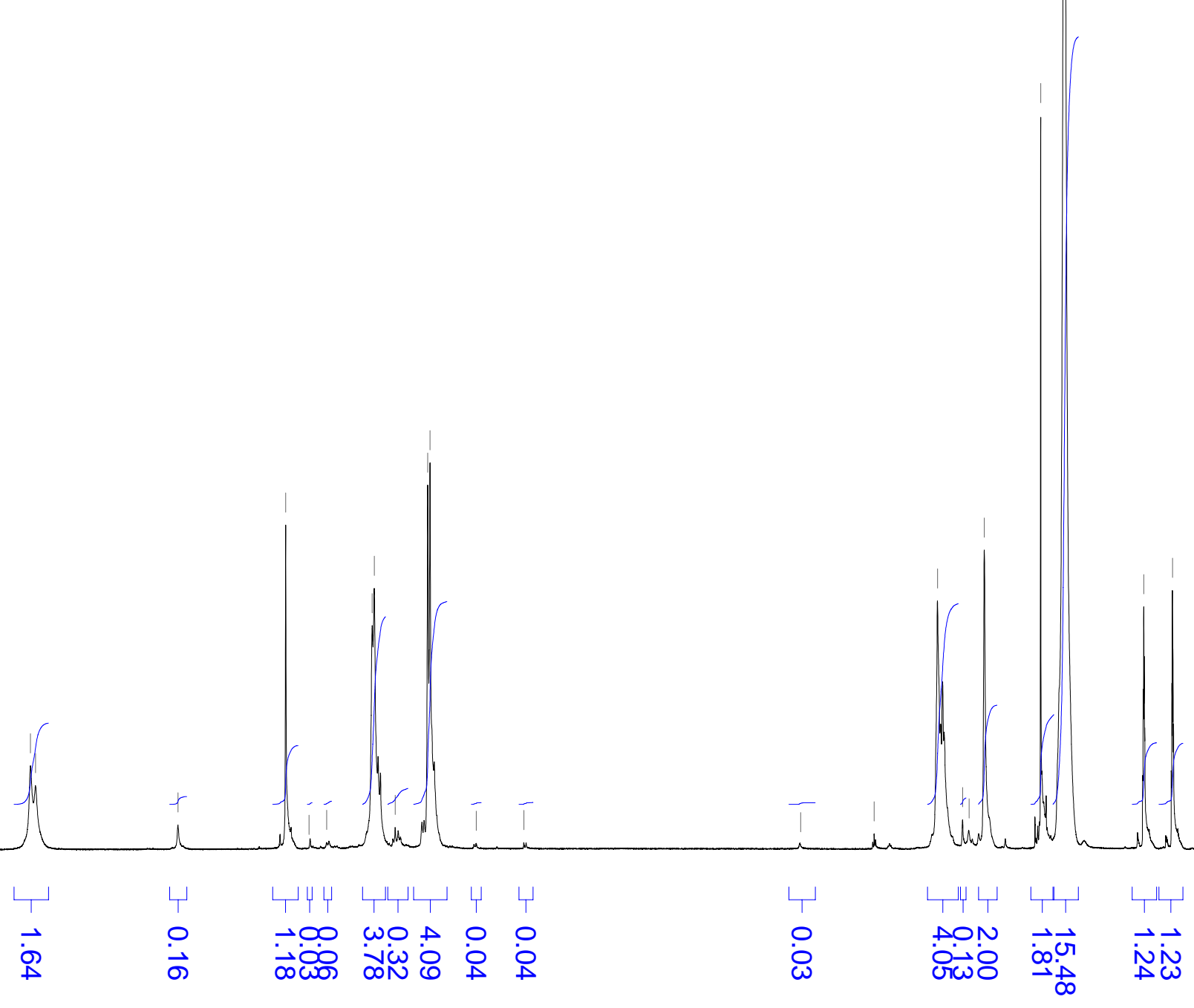

$\begin{array}{ll}1 & 0 \\ \text { जั } & 0 \\ \infty & \infty\end{array}$

4
0
0
0

$\begin{array}{lll}10.0 & 9.0 \quad 8.0\end{array}$
7.0

6.0

5.0

4.0

3.0

2.0

1.0

0.0 


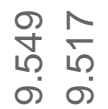

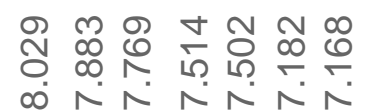

Figure S7: $600 \mathrm{MHz}{ }^{1} \mathrm{H}$ NMR Spectrum of MP6 in DMF- $\mathrm{d}_{6}$.

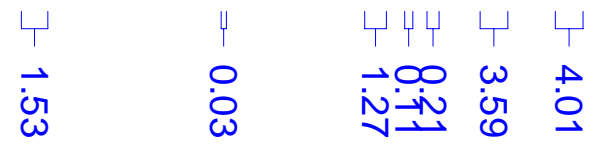

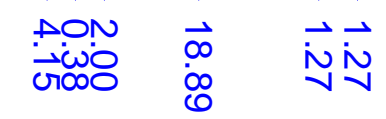

$\stackrel{\circ}{\perp}$

\section{$\begin{array}{llll}10.0 & 9.0 & 8.0 & 7.0\end{array}$}

6.0

5.0

4.0

3.0

2.0

1.0

0.0 


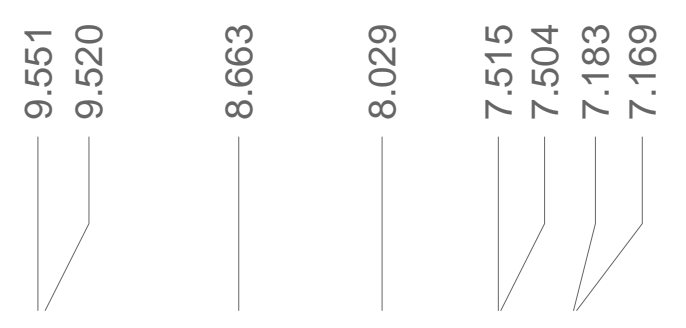

Figure S8: $600 \mathrm{MHz}{ }^{1} \mathrm{H}$ NMR Spectrum of MP7 in DMF- $\mathrm{d}_{6}$.
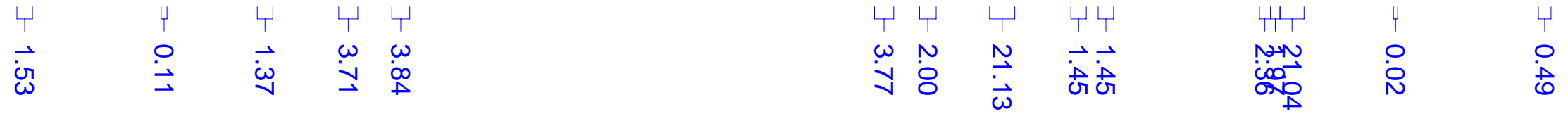

\section{$10.0 \quad 9.0$} 8.0 7.0

6.0

5.0

4.0

3.0

2.0

1.0

0.0 


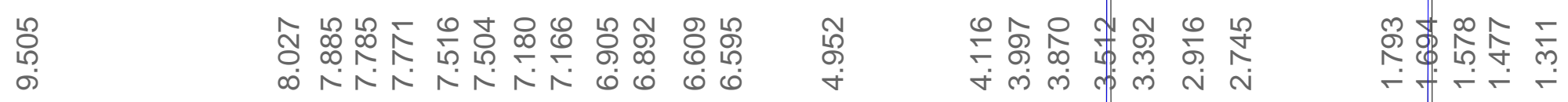

Figure S9: $600 \mathrm{MHz}{ }^{1} \mathrm{H}$ NMR Spectrum of SP1 in DMF- $\mathrm{d}_{6}$.

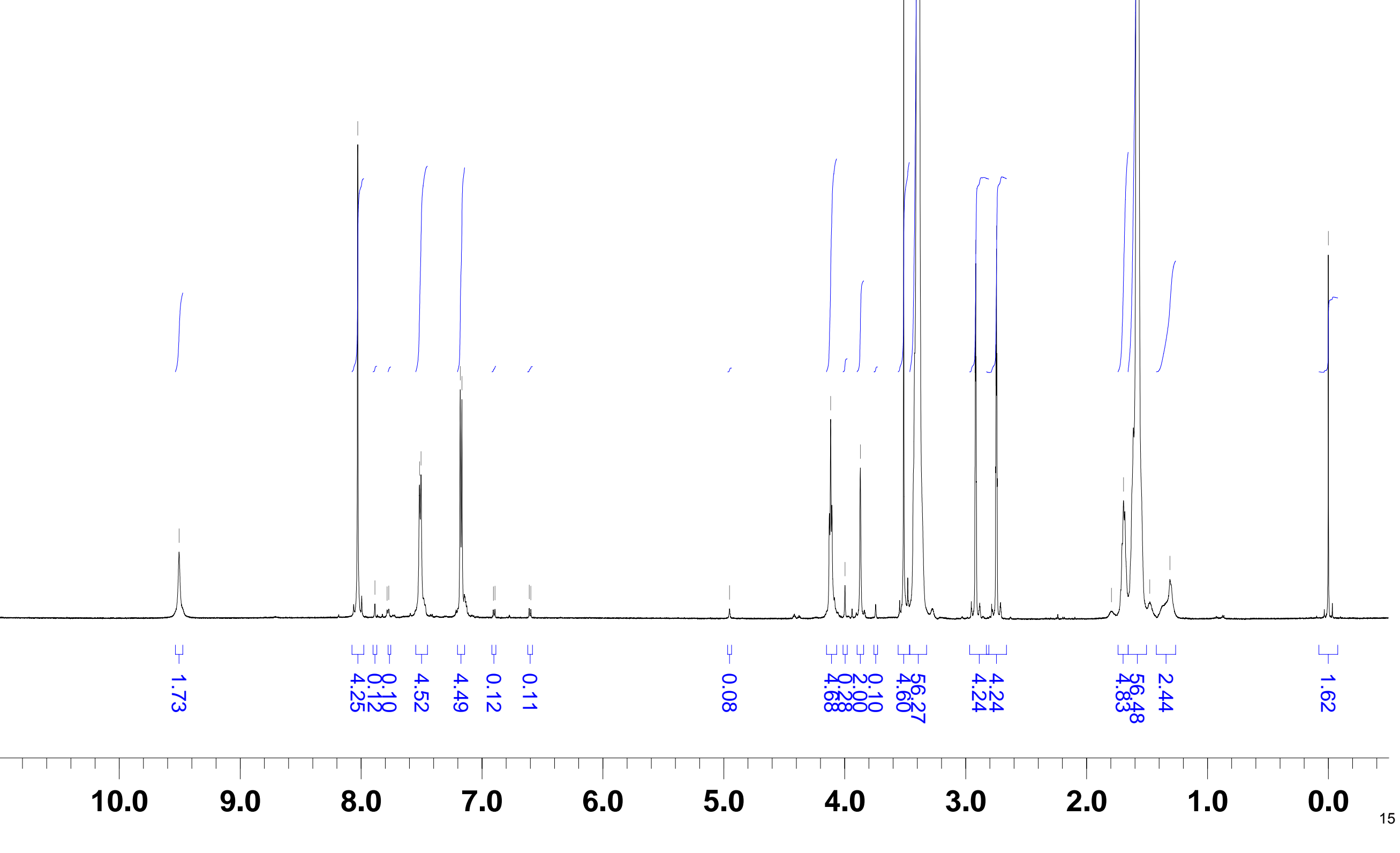


Figure S10: $600 \mathrm{MHz}{ }^{1} \mathrm{H}$ NMR Spectrum of SP2 in DMF- $\mathrm{d}_{6}$.
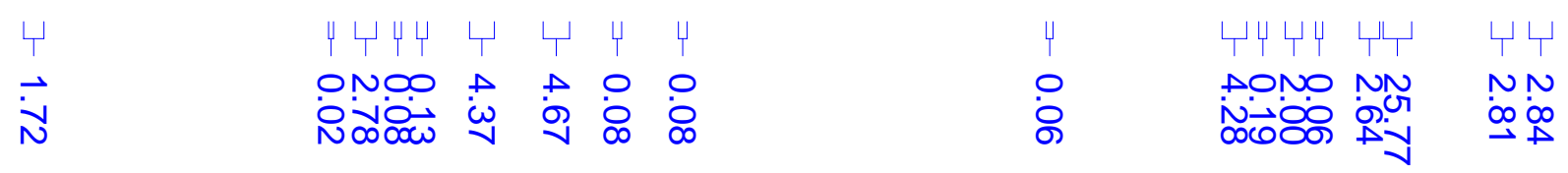

ํํํ

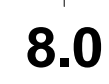

7.0

6.0

5.0

4.0

3.0

2.0

1.0

0.0 

商昜

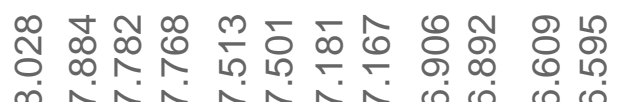

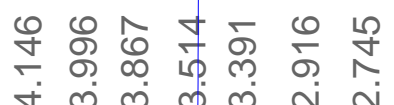

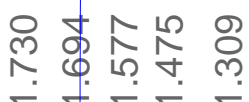

8
8
0

Figure S11: $600 \mathrm{MHz}{ }^{1} \mathrm{H}$ NMR Spectrum of SP3 in DMF- $\mathrm{d}_{6}$.

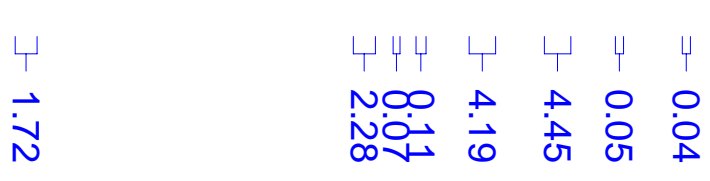

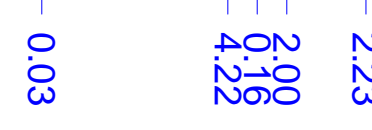

$\stackrel{\substack{N \\ \mathbb{N}}}{\mathbb{N}}$

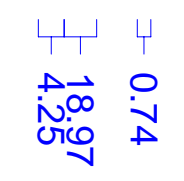




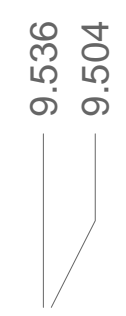

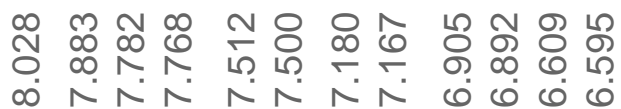

Figure S12: $600 \mathrm{MHz}{ }^{1} \mathrm{H}$ NMR Spectrum of SP4 in DMF- $\mathrm{d}_{6}$.
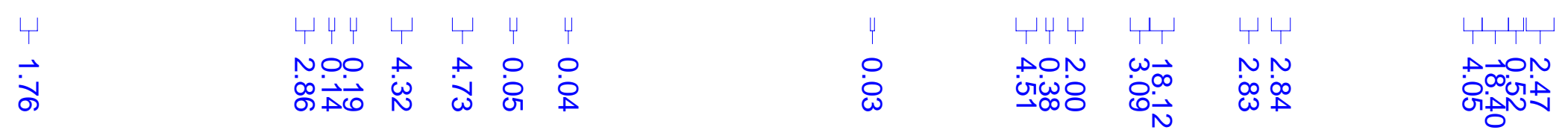

量善

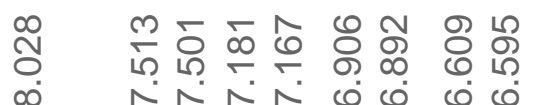

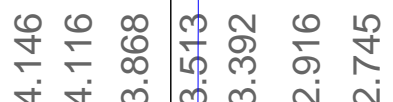

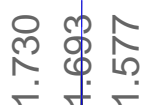

Figure S13: $600 \mathrm{MHz}{ }^{1} \mathrm{H}$ NMR Spectrum of SP5 in DMF- $\mathrm{d}_{6}$.

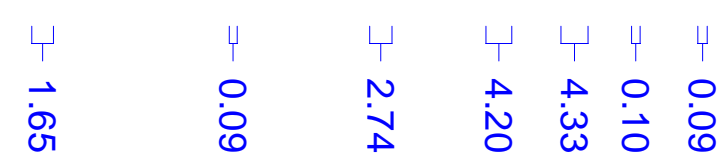

$\stackrel{0}{9}$

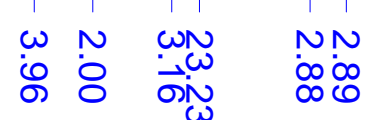

Do

$\triangle N$

:

1
4

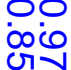

10.0

9.0

8.0

7.0

6.0

5.0

4.0

3.0

2.0

1.0

0.0

19 
a

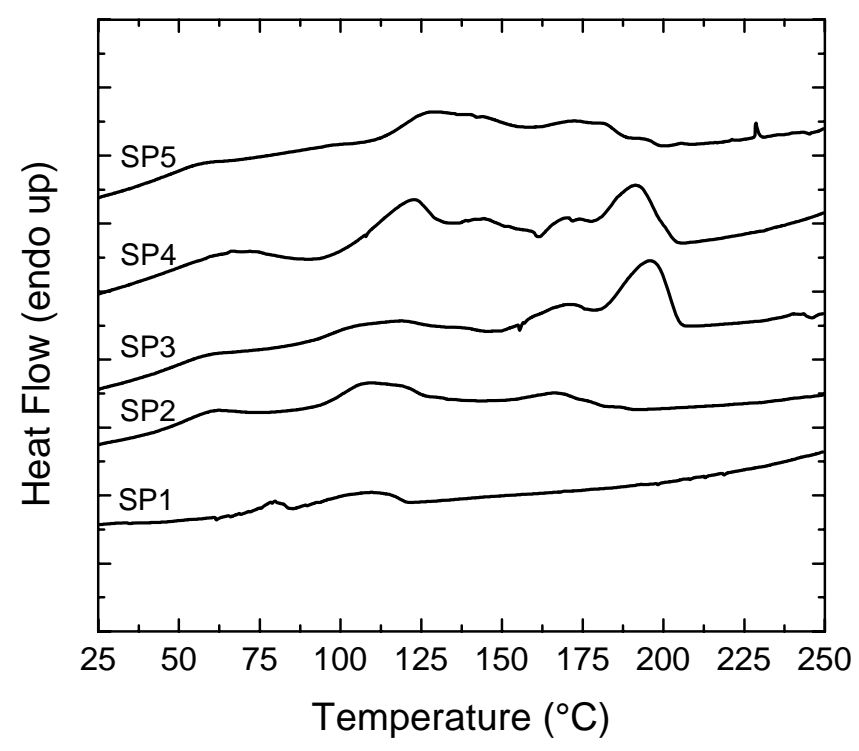

b

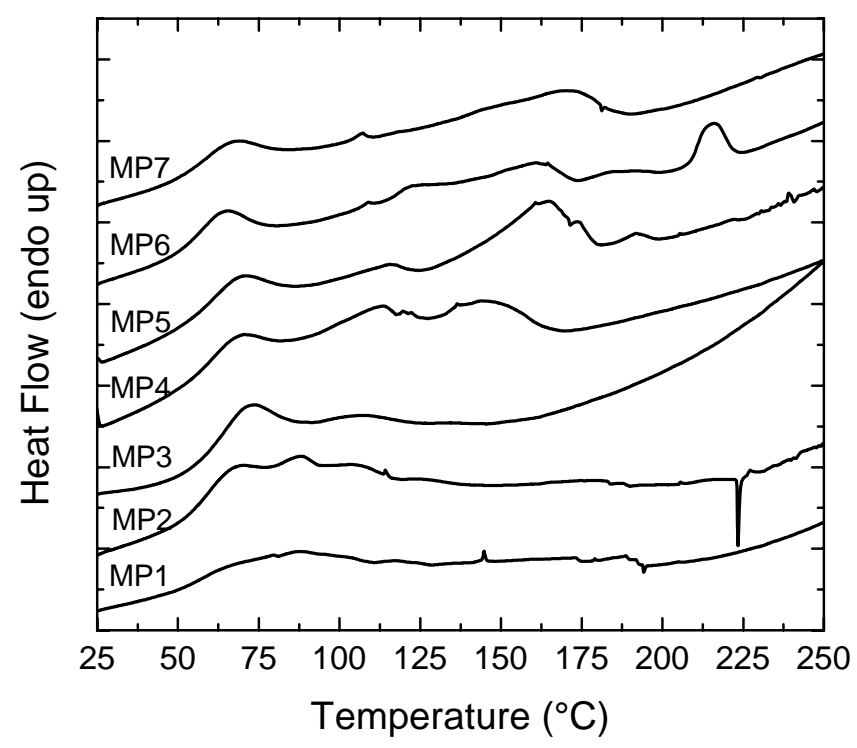

Figure S14. DSC traces showing the first heating of SP1-SP5 (a) and MP1-MP7 (b). All experiments were conducted under $\mathrm{N}_{2}$ at a heating rate of $10^{\circ} / \mathrm{min}$. 


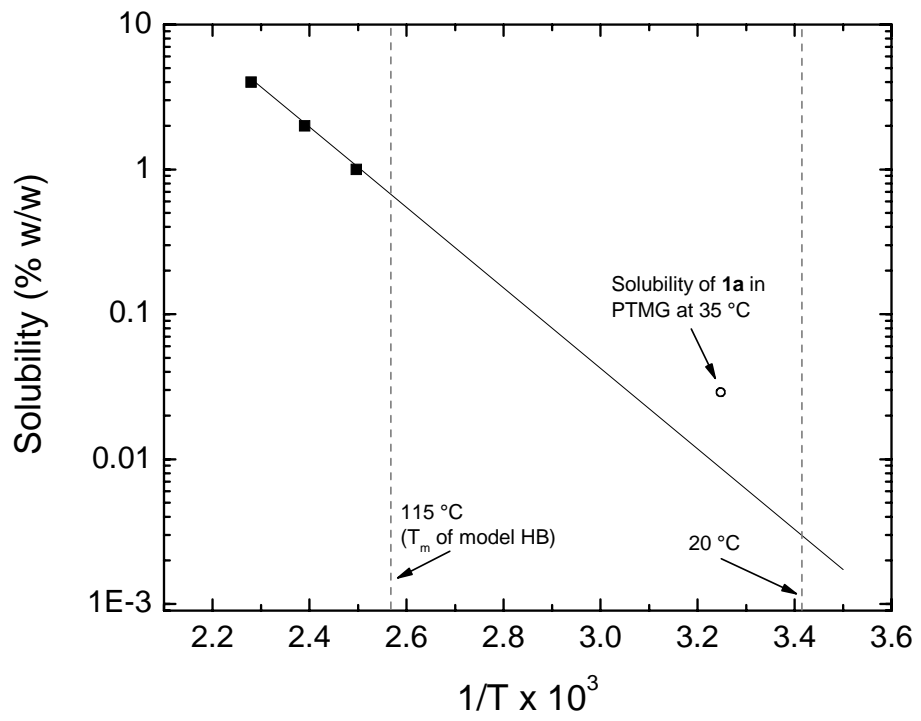

Figure S15. Solubility of 1a in dibutyl-4,4'-methylenebis(phenylcarbamate) (BMCP, - $)$ and PTMG (०). The solid line is a least squares fit to solubility data obtained for BMCP and extrapolated to room temperature. The dashed vertical lines indicate the melting temperature of BMCP $\left(115^{\circ} \mathrm{C}\right)$ and room temperature $\left(20^{\circ} \mathrm{C}\right)$. The solubility of 1c in BMCP was determined in the temperature regime between 115 and $170{ }^{\circ} \mathrm{C}$ using polarized optical microscopy. Between 1 and $4 \% \mathrm{w} / \mathrm{w}$ 1c was dissolved in DMPC at $200{ }^{\circ} \mathrm{C}$ and cooled to $90{ }^{\circ} \mathrm{C}$ where BMCP had crystallized and large-scale phase separation between the dye and BMCP was observed. The temperature was then increased at a rate of $10^{\circ} / \mathrm{min}$. The samples were inspected in a microscope and the temperature at which the dye crystals had completely dissolved corresponded to the dissolution temperature. Solubility of 1c in PTMG was determined by placing $5.2 \mathrm{mg}$ of $\mathbf{1 c}$ in a flask kept at $35^{\circ} \mathrm{C}$. PTMG was added incrementally until the dye had completely dissolved. 


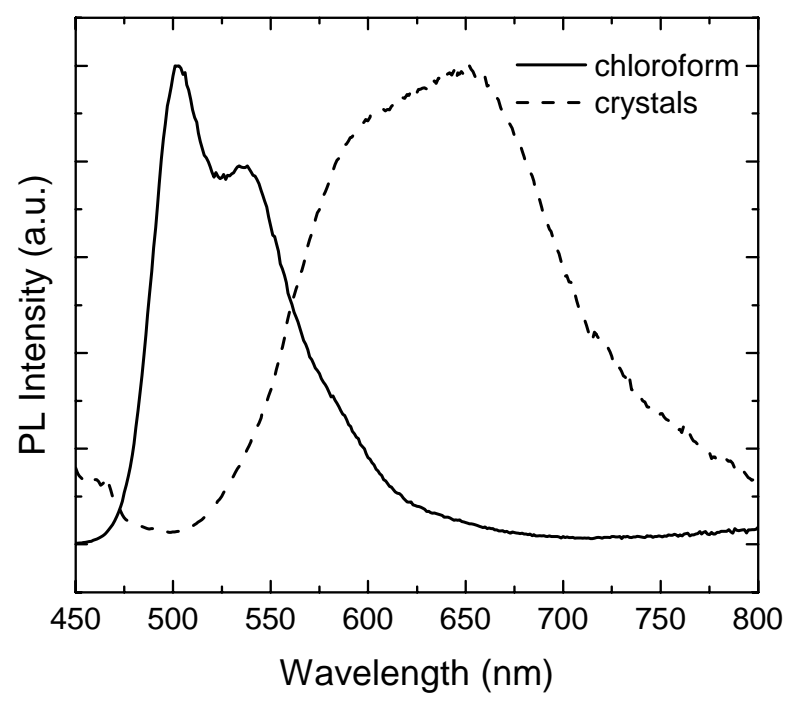

Figure S16. Normalized PL emission spectra of a chloroform solution and "as synthesized" crystals of 1c. 


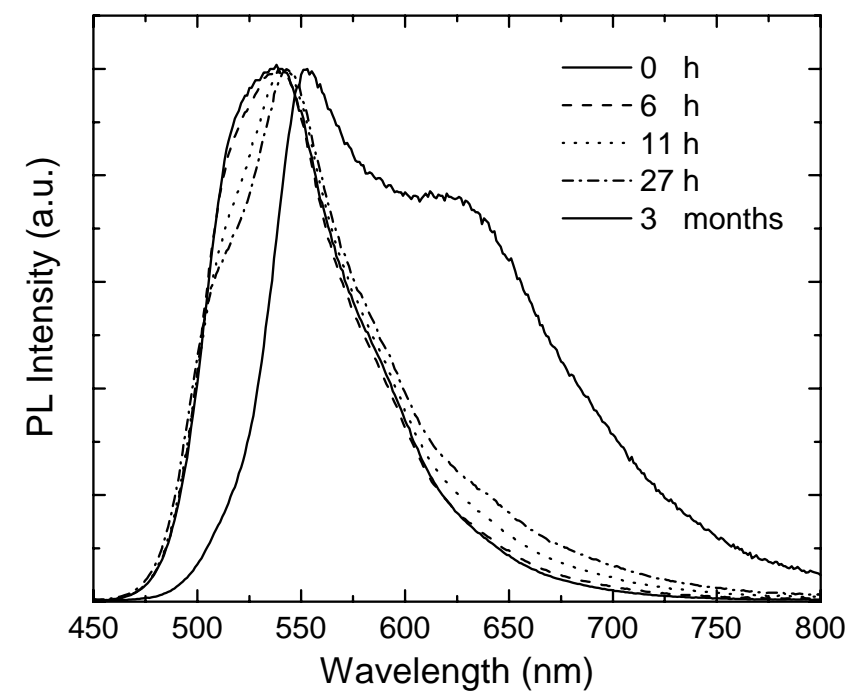

Figure S17. PL emission spectra of a blend film of Texin 985 and $0.2 \% \mathrm{w} / \mathrm{w}$ 1a cooled slowly to room temperature from the melt $(15 \mathrm{~min})$ as function of time after cooling. 
a

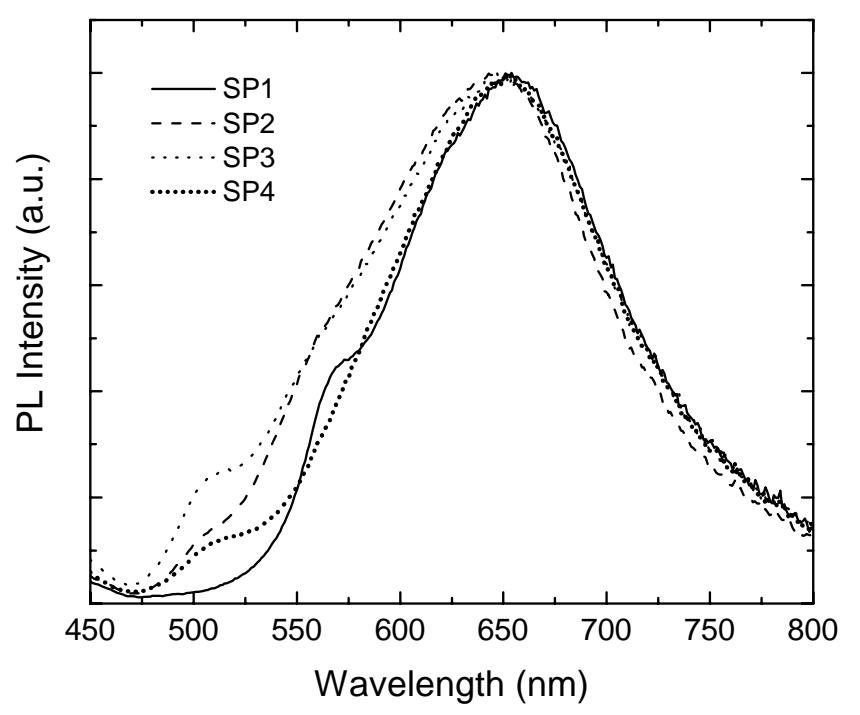

b

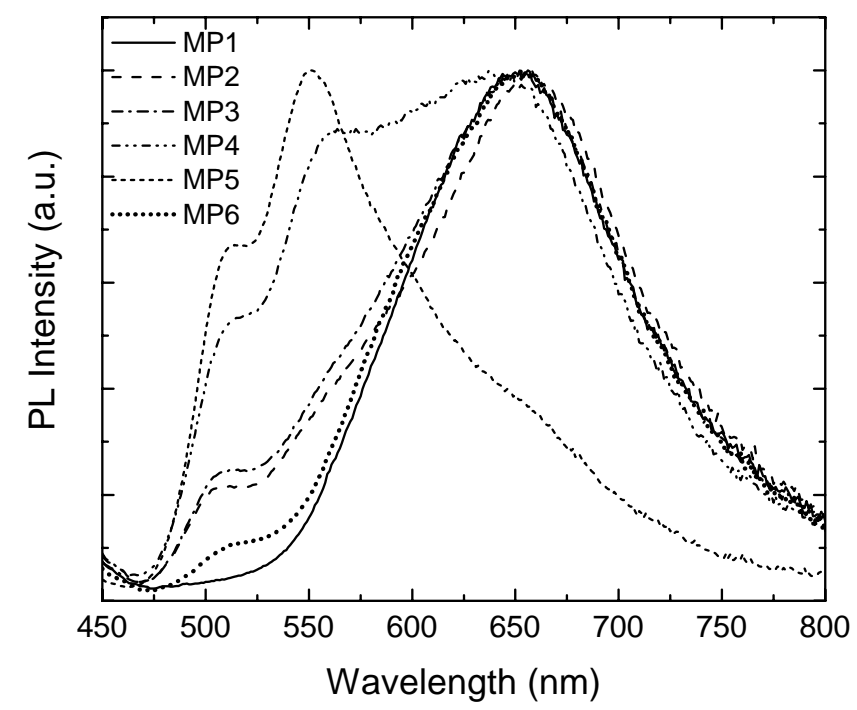

Figure S18. PL emission spectra of a) SP1-SP4 after storage at ambient for 4 months; b) MP1-MP6 after storage at ambient for 10 months. 